\title{
Elaboración y aplicación de un proyecto interdisciplinar en las etapas de Infantil y Primaria sobre prevención de accidentes promovido desde el Área de Educación Física \\ Development and application of an interdisciplinary project on accident prevention promoted from the Physical Education Area in the stages of Early Childhood and Primary Education \\ *Lucía Peixoto-Pino, *Javier Rico-Díaz, **Víctor Arufe Giráldez \\ *Universidad de Santiago de Compostela (España), **Universidad A Coruña (España)
}

Resumen. El currículo de Educación Infantil y el de Educación Física en la etapa de primaria, promueve contenidos relacionados con la prevención de accidentes y los primeros auxilios (PPAA). El enfoque globalizador y los proyectos interdisciplinares se han mostrado como efectivos y pedagógicamente recomendados. El área de Educación Física tradicionalmente es la gran dinamizadora de los centros educativos. En este artículo se aborda un proyecto institucional en el marco de un contrato programa con la Consellería de Cultura, Educación y Ordenación Universitaria de la Xunta de Galicia, desarrollado en un CEIP de la provincia de Pontevedra, en el que se realizaron 13 actividades globales, en la que participaron 30 docentes y que pudo llegar a 430 alumnos. Esta experiencia muestra los puntos clave para la organización e implementación de un proyecto interdisciplinar que abarque un curso lectivo y que pueda servir como referente para otras iniciativas lideradas por los maestros de Educación Física. Demuestra que un claustro de maestros, coordinado y con iniciativa pedagógica común puede organizar e implementar un proyecto interdisciplinar sobre una temática educativa curricularmente reconocida y socialmente necesaria. Se observa también la importancia de implicar a toda la comunidad educativa para que este tipo de acciones repercuta en un proceso de enseñanza-aprendizaje más reflexivo y eficiente.

Palabras clave. Proyecto interdisciplinar, educación física, competencias, prevención accidentes, proceso de enseñanza-aprendizaje.

Abstract. The curricula of Early Childhood Education and Physical Education in the primary stage promote content related to accident prevention and first aid (PPAA). Globalizing approaches and interdisciplinary projects have been shown to be effective and pedagogically recommended. The area of Physical Education traditionally assumes the highest dynamic role within educational centers. This article deals with an institutional project within the framework of a program funded by the Department of Culture, Education, and University Planning of the Xunta de Galicia, developed in a CEIP in the province of Pontevedra In the mentioned project, 13 global activities were carried out, with 30 teachers and 430 students being engaged in. This experience shows the key points for the organization and implementation of an interdisciplinary project covering a school course, which could serve as a reference for other initiatives led by Physical Education teachers. It shows that coordinated faculty members with common pedagogical initiative can organize and implement an interdisciplinary project on a curricularly recognized and socially necessary educational subject. We also observe the importance of involving the entire educational community so for this type of actions to trigger a more reflective and efficient teaching-learning process.

Key words. Interdisciplinary project, physical education, competencies, accident prevention, teaching-learning process.

\section{Introducción}

El área de Educación Física reúne una serie de características interdisciplinares con otras materias y temáticas curriculares (Díaz, 2010). El enfoque actual del marco curricular, basado en las competencias y los contenidos (Gómez etal., 2008) promueveun aprendizaje integrado y significativo. Los proyectos interdisciplinares que promueven valores, hábitos y competencias han sido tratados tanto en las etapas de primaria y secundaria en numerosas experiencias reales (Jorquera, Leiva, Molina, \& Sánchez, 2018; Castellar, Pradas, Rapún, Coll, \& Pérez, 2013; PeixotoPino, 2015a). En este sentido es la etapa de primaria la que presenta el mayor número de experiencias y actividades para la prevención de accidentes y en implementación de experiencias sobre primeros auxilios (Lago-Ballesteros, Basanta-Camiño, \& Navarro-Patón, 2018). Dehecho, la propia legislación educativa promueve desde el área de Educación Física tanto en educación infantil como en educación primaria («Real Decreto 126/2014, de 28 de febrero, por el que se establece el currículo básico de la Educación Primaria.», s.f.; «Real Decreto 1630/2006, de 29 de diciembre, por el que se establecen las enseñanzas mínimas del segundo ciclo de Educación infantil.», s.f.). Los maestros en general y los de Educación Física en particular, son profesionales capacitados docentemente para conducir un programa de enseñanza/aprendizaje efectivo en el ámbito de la prevención de accidentes (Pichel-López et al., 2017) y las recomendaciones de las sociedades científicas avalan la iniciativa para promover estos contenidos desde la escuela (Bohn, Lukas, Breckwoldt, Böttiger, \& Van Aken, 2015; Greif et al., 2015).

El sistema educativo actual busca dar respuesta a los problemas sociales y, los accidentes infantiles son una de las principales causas de muerte en los países desarrollados (World Health Organization, 2008).

Fecha recepción: 16-09-18. Fecha de aceptación: 02-10-18 Víctor Arufe Giráldez varufe@yahoo.es
Desde hace más de una década, los proyectos interdisciplinares se han instaurado en los centros educativos, promoviendo la conexión de contenidos y fomentando la adquisición de competencias, frente al enfoque tradicional atomizado en asignaturas aisladas (Díaz, 2010).

Por tanto, el objetivo de este artículo es dar a conocer desde una perspectiva docente y real, una propuesta de trabajo interdisciplinar desarrollada en un centro educativo público dela provincia de Pontevedra.

\section{Metodología}

\section{Participantes y localización}

Un total de 430 estudiantes del CEIP Mestre Ramiro Sabell (Ponteareas - Pontevedra) participaron en este proyecto, enmarcado dentro de las actividades propuestas por el centro docente, recogidas en el proyecto educativo (PE) y aprobadas por el Consejo Escolar, con la participación de la comunidad educativa.

\section{Diseño del programa}

«TU PUEDES SALVAR UNA VIDA» es un proyecto recogido en el contrato programa de centro para la mejora del éxito escolar de la Xunta de Galicia («mejora de la convivencia»). Esto significa, que el enfoque del mismo recogido en la Consellería de Cultura, Educación y Ordenación Universitaria se centra en la consecución de la disminución de conflictos e incidentes de mal comportamiento en el alumnado. El tema de la prevención de accidentes y primeros auxilios resultó muy adecuado para abordarlo puesto que el enfoque planteado desde un principio fue que la mejora de la convivencia, el cumplimiento de las normas cívicas y el respeto a los demás es un punto clave para la prevención de riesgos.

Detectar el peligro, evitarlo y, en caso de accidente, actuar con la mayor rapidez posible es de vital importancia para salvar una vida.

La pretensión de este programa además del meramente conceptual y procedimental, fue que los alumnos reconociesen sus sentimientos y 
sus emociones, las identificasen y fuesen quienes de manejarlas sobre todo en situaciones de estrés y alerta. De forma paralela se trabajó el reconocimiento de sus propios límites y posibilidades, haciendo hincapié en la importancia de sus actos a la hora de provocar o prevenir accidentes y resaltando la importancia de la toma de decisiones correctas a la hora de actuar ante un accidente. Se afianzaron estos contenidos con la pretensión de que el alumnado hiciese de la prevención una verdadera forma de ser y de vivir.

El programa fue dirigido desde el área de Educación Física y desde la coordinación de actividades complementarias, dentro del equipo de dinamización de la convivencia y en relación directa con la jefatura de estudios, dirección y diversos equipos de trabajo (Tecnologías de la Información-TICs-, Bibliotecay Equipo de Normalización Lingüística).

Al ser un proyecto interdisciplinar estuvo implícito en la mayoría de las áreas, sirviendo como hilo conductor para la mayor parte de las actividades complementarias, recayendo en toda la comunidad educativa (estuvo implicada la totalidad del profesorado y alumnado, conserje y familias que colaboraron en la realización de diversas actividades).

\section{Objetivos curriculares.}

Este proyecto se fundamenta en la Ley Orgánica 2/2006, de 3 de mayo, de Educación, que es la ley en vigor en todos los cursos de Educación Infantil (EI) y Educación Primaria (EP) en la fecha de realización del proyecto. La LOE en su capítulo III donde determina el currículo, establece el método por proyectos como una fórmula muy adecuada para alcanzar el desarrollo de las competencias básicas.

A partir de aquí, se establecen los siguientes objetivos para abordar en el proyecto (Peixoto-Pino, 2015b):

- Reforzar conocimientos y destrezas en las áreas instrumentales.

- Mejorar los resultados académicos.

- Favorecer las relaciones entre las familias y el centro.

- Mejorar su integración social en el grupo y en el centro.

- Prevenir los conflictos detectando las emociones, las necesidades vitales así como los sentimientos propios y los de los demás.

- Detectar los peligros, aprender a prevenirlos y actuar acertadamente en caso de accidente a través de las actividades y conmemoraciones en el centro.

- Tomar conciencia de los peligros que ocasionan ciertas actividades, adoptando una actitud de respeto y cuidado del propio cuerpo y el de los demás miembros del grupo.

- Ser consciente de la importancia de la colaboración con el grupo en caso de accidente.

- Fomentar el trabajo interdisciplinar en todas las etapas educativas de nuestro centro (Educación Infantil y Educación Primaria).

- Propiciar entornos de trabajo colaborativo entre alumnos y docentes.»

\section{Cronograma}

El presente proyecto se realizó durante el curso 2012/13. En la Figura 1 se resume la temporalización de las actividades durante el año académico, con cuatro bloques de tareas que se desarrollarían de forma permanente (Concurso mascota, Conmemoraciones, Trabajo de áreas, Formación del profesorado) y numerosas fechas conmemorativas con actividades vinculadas a cada evento: Declaración Mundial de los Derechos Humanos, Día de la Paz, Seguridad Vial, Carnaval Preventivo, Semana de la Prensa, Día de la Mujer Trabajadora, Día de los Derechos del Consumidor, Día Mundial de la Salud, Día de Europa, Dia Mundial del Medio Ambiente.

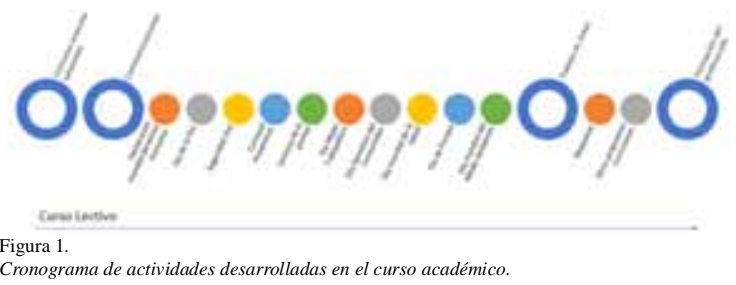

\section{Actividades}

Actividad global 1. Concurso de la mascota «el salvavidas»

A modo de actividad introductoria se propuso al alumnado la creación de una mascota que representase el espíritu del proyecto. Esta mascota se elaboraría a lo largo de todo el curso escolar, pero la presentación de la idea desde el primer momento buscaba fomentar la curiosidad y también darles participación a las familias (figura 2).

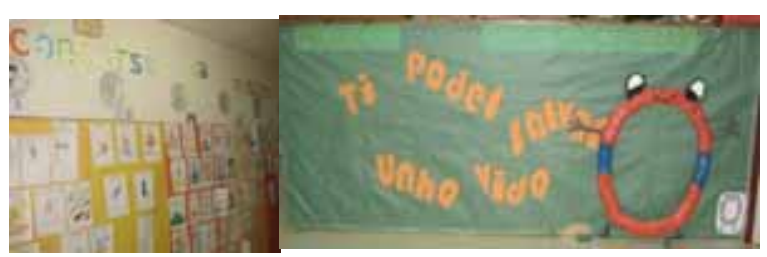

Figura 2.

Murales del concurso de mascota "salvavidas".

Actividad global 2. Conmemoraciones

Día de la Paz y Día de la Declaración Universal de los Derechos Humanos

En diciembre, coincidiendo con el Día de la declaración Universal de los Derechos Humanos (10 diciembre,) se comenzaron a trabajar en las aulas los mensajes de prevención de riesgos repartidas en diversas profesiones que se expusieron finalmente en el mural del día de la Paz (figura 3).

Se elaboraron dos murales, uno para el edificio de Educación Infantil (E.I.) y otro para el de Educación Primaria(E.P.) en los que se plasmaron los dibujos y mensajes relacionados con la prevención de accidentes titulados «Mejorando la convivencia evitamos riesgos». En ellos cada aula elaboró un mensaje de prevención relacionado con los diferentes peligros a los que los alumnos/as tienen más acceso (ahogamientos, electricidad, educación vial...) Esos compromisos fueron la base del manifiesto de paz leído en el acto de conmemoración. Otro aspecto relevante de esta conmemoración fue la lectura de un manifiesto cuya finalidad, además de dar a conocer y practicar el plan de evacuación necesario por si ocurriese algún accidente, era hacer conscientes a los niños de la importancia del cumplimiento de las normas cívicas y la ayuda hacia los demás cómo medio de prevención de accidentes. Otra actividad realizada durante esta conmemoración fue la puesta en práctica del plan de evacuación con la ayuda de los bomberos. Esta simulación fue una sorpresa para todo el alumnado.

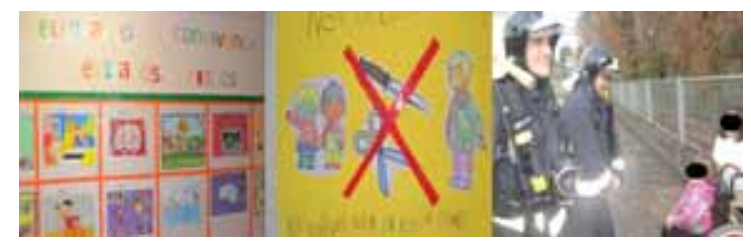

Figura 3.

Actividades Día de la Paz y del Día de la Declaración de los Derechos Humanos.

Visita de la Dirección General de Tráfico (DGT)

El alumnado recibió la visita de dos agentes de tráfico, los cuáles les enseñaron las normas de circulación, así como las conductas peatonales básicas. El alumnado acudió al centro con bicicletas, patines, patinetes y cars (cedidos por tráfico) y siempre con casco para participar en el recorrido de educación vial que la DGT les preparó (figura 4).

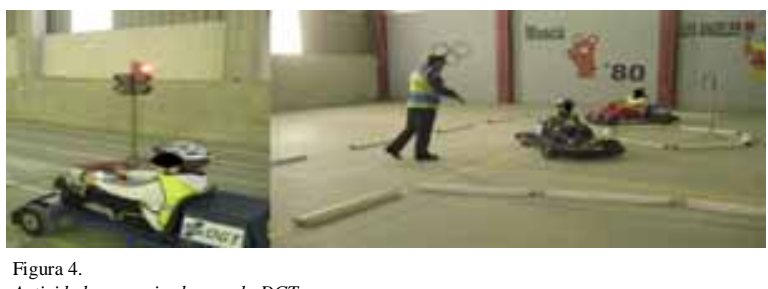

Actividades organizadas por la DGT.

Carnaval preventivo

El claustro de profesorado decidió un organizar un carnaval temático, basado en la prevención o ayuda a los demás. Cada ciclo/curso tenía que 
buscar una temática que lucirían en el desfile de carnaval:

- E.I.: Superhéroes.

- Primero E.P.: Técnicos en emergencias sanitarias (TES).

- Segundo E.P.: Policías.

- Tercero y cuarto E.P.: Rescatadores de montaña.

- Quinto y sexto: Médicos/Enfermería.

\section{Semana de la prensa}

A lolargo del curso cada aula (una por semana) analizaba las noticias relacionadas con los accidentes y estudiaban cómo se podrían haber evitado, si fuera el caso. Cada aula elaboró un mural con las noticias de los periódicos de esa semana en torno al tema de los accidentes; convirtiéndose así en «reporteros de sucesos». Esos murales estuvieron en los pasillos del colegio y en la semana de la prensa grabamos por aulas las noticias en el ordenador en el aula de informática y las colgamos en la radio escolar de la página web (trabajo realizado por el equipo de las TICs del colegio).

\section{Día internacional de la mujer trabajadora}

Se analizaron en las aulas cuáles son las profesiones relacionadas con el salvamento y prevención de riesgos predominantementefemeninas o masculinas: bomberos, enfermeras, policías, médicos, socorristas...

\section{Día mundial de los derechos del consumidor}

Se trató en las aulas sobre la calidad de la sanidad y el buen uso de ellay del número de emergencias 112.

\section{Día Mundial de la salud}

Se comenzó ese día con la visualización de los capítulos del proyecto educativo de la Xunta de Galicia «E Por qué?» https://www.edu.xunta.es/ espazoAbalar/sites/espazoAbalar/files/datos/1303196843/contido/ e_por_que/eporque2.html (de infantil a segundo ciclo de educación primaria) y lecturas sobre los Primeros Auxilios (PPAA) básicos (para el tercer ciclo de primaria). Con estos datos elaboraron unos murales gigantes (figura 5) repartidos por cursos sobre los accidentes más relevantes (fuego, elementos cortantes, atragantamientos, RCP básica, protocolo de actuación ante un accidente, intoxicaciones y heridas).

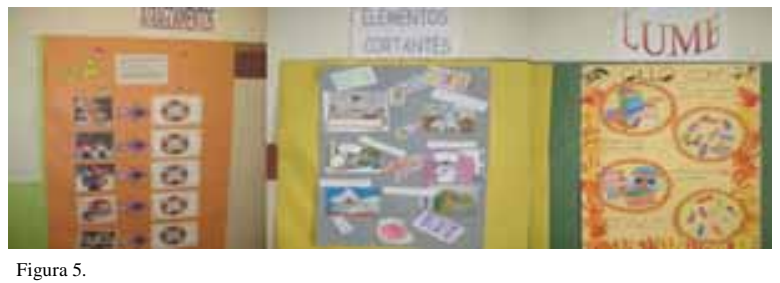

Actividades Día Mundial de la Salud.

\section{Día de Europa}

Analizaron los distintos sistemas de salud existentes en Europa. Se repartió un documento en el que se explicaban los dos modelos sanitarios principales en la UE. Se hizo una lectura comprensiva con actividades posteriores relacionadas con la localización en un mapa de los países que tenían cada uno de esos modelos y un test sobre el número europeo de emergencias 112.

\section{Día mundial del medio ambiente}

Contando con la colaboración de la DGT, policía local, protección civil y el club ciclista Ponteareas organizaron una marcha ciclista al campo de fútbol municipal. El motivo principal de esta marcha era que los alumnos conociesen y vivenciasen las medidas de seguridadnecesarias para las salidas en bicicleta por carretera. En la localidad donde se encuentra el colegio hay mucha tradición ciclista, siendo la cuna de grandes ciclistas, como el ganador de la Vuelta Ciclista a España, Álvaro Pino, por lo que se consideró un tema de especial importancia. Una vez en el campo de fútbol realizaron un recorrido de actividades y juegos de PPAA: buscadores de riesgos, recorrido de educación vial, taller de RCP, SOS expresivo y SOS memory y patrullando la ciudad (figura 6).

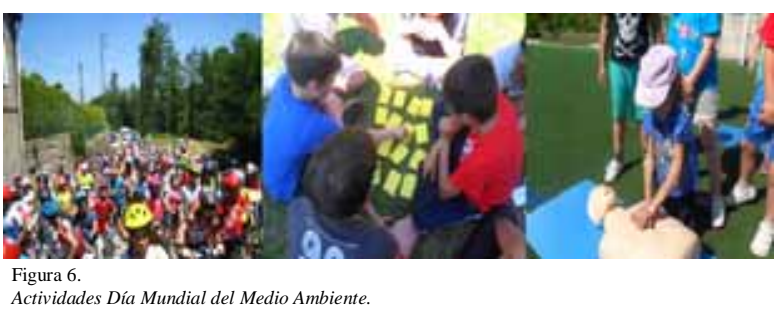

Actividad global 3. Trabajo en áreas específicas y en el entorno familiar

Para un enfoque global y transversal, cada área debería reforzar con actividades propias el proyecto común. Por tanto la prevención y PPAA, todas ellas recogidas en las programaciones de aula de los diferentes cursos. Cada maestro trabajó el tema en aquellas disciplinas en las que consideró más oportuno hacerlo tomando, en todo momento, el tema como hilo conductor de su programación anual. Entre otras propuestas, destacaron:

- Matemáticas: mediante el recuento y posterior cálculo estadístico que muestra el porcentaje de riesgos en los hogares de nuestro alumnado.

- Lengua castellana y lengua gallega: trabajaron la comprensión lectora a través de libros cedidos por la fundación Mapfre con actividades y preguntas sobre la lectura.

- Resumen y esquemas de temas relacionados con la prevención y PPAA que después plasmaron en murales gigantes en los pasillos.

- Plástica: elaboración de los murales, decoración del centro, elaboración de los disfraces, realizaciones de maquetas preventivas con la ayuda de las familias...

- Inglés: trabajo de una Unidad Didáctica acerca de los peligros en las playas y carreteras.

- Educación física: con una unidad didáctica de PPAAen las que los niños de $1^{\circ}$ ciclo aprendían a distinguir riesgos a través de un cuento motor y otras actividades de vuelta a la calma y los niños del segundo y tercero ciclo aprendían las técnicas básicas de PPAA(atragantamientos, posición lateral de seguridad, RCP básica...).

- Conocimiento del medio: estudiando los diferentes sistemas de salud de los países de UE (actividad hecha el día de Europa).

- Biblioteca: construcción de la «esquina de prevención» en el que recopilamos libros y revistas relacionadas con el tema. Colgamos preguntas en el blog de la biblioteca «lunares habla» alrededor del tema de la prevención.

Para que el proyecto fuese realmente interdisciplinar, el entorno familiar del niño juega un papel clave, por tanto, varias actividades fueron propuestas para realizar con sus padres y madres.

Elaboración de maquetas sin riesgos: en colaboración con las familias pedimos al alumnado de $6^{\circ}$ E.P. que elaboraran una maqueta relacionada con el tema del proyecto (hospitales, playas con socorristas, accidentes de tráfico, bomberos, hospitales de campaña, etc...). Estas maquetas decoraron la entrada del colegio durante lo que duró el proyecto (figura 7).

Asimismo se repartió un cuadro de recogida de datos en el que tenían que marcar, en sus casas y en colaboración con su familia con un simple SÍ o NO si habían sufrido algún accidente con alguno de los temas señalados. Se convirtieron por un día en «Inspectores de riesgos del hogar». Con estos datos calcularon el porcentaje de prevalencia de unos accidentes sobre otros quedando plasmado en un gráfico gigante en uno de los pasillos del centro (figura 7).

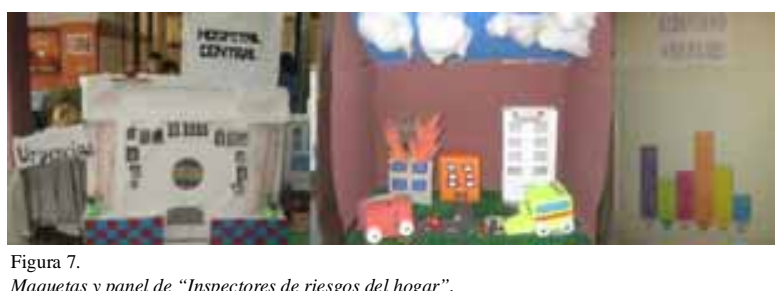

Maquetas y panel de "Inspectores de riesgos del hogar". 


\section{Actividadd global 4. Formación del profesorado}

Los docentes del centro, así como su personal de servicios tuvieron la oportunidad de participar en una actividad formativa y voluntaria sobre actitudes para la prevención y PPAA impartido por dos expertos universitarios, especialistas en la temática. Este curso tuvo como finalidad ofrecer herramientas didácticas así como dotar de habilidades para la enseñanza de la prevención de accidentes desde la escuela y la intervención básica en caso de emergencia.

\section{Resultados}

En la tabla 1 se resumen los principales resultados cuantitativos del programa. Un total de 13 actividades globales y coordinadas fueron realizadas en el curso lectivo en un claustro compuesto por 30 docentes. Esto supone una media de dos actividades al mes. El número de acciones directas sobre el alumnado desde las diferentes áreas fue de 3250. El proyecto fue evaluado positivamente por la Consellería de Cultura, Educación y Ordenación Universitaria de la Xunta de Galicia.

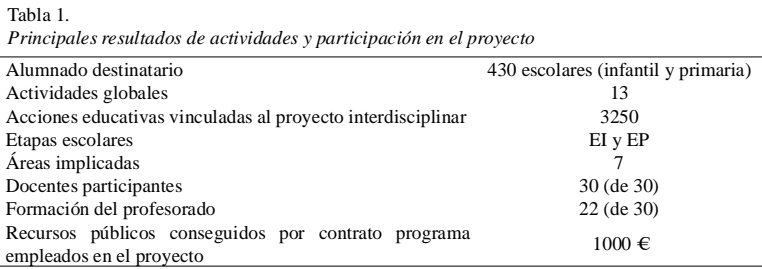

Analizando los resultados curriculares, cabe destacar que, en las incidencias de comportamiento, se produjo una disminución de los registros en relación a los cursos anteriores. Además, cabe destacar la consecución un ambiente motivador, no solo en las actividades complementarias en las que estuvo presente, sino en las diversas áreas en las que al trabajar alrededor de un tema de interés como son los primeros auxilios, aumentaron la motivación en ciertas actividades curriculares (lectura, plástica, búsqueda de información a través de soporte informático...) planteadas por el profesorado en las diversas áreas.

Desde el punto de vista de la evaluación formal, la participación en los proyectos así como sus implicaciones académico-curriculares, fueron evaluadas por cada tutor o docente especialista, mediante los instrumentos de registro habituales y referidos en la programación docente (listas de control y/o diario de aula), fomentándose desdeel claustro de profesorado, una evaluación formativa, continua y participativa.

\section{Discusión}

El objetivo de este trabajo fue diseñar y aplicar un proyecto interdisciplinar para favorecer la adquisición de conductas responsables y conocimientos básicos de PPAAdesde la etapa infantil hasta el último curso de primaria. Los proyectos interdisciplinares a nivel escolar, y con la promoción del área de Educación Física en particular, contribuyen a la interrelación de contenidos (Díaz, 2010), promoviendo aprendizajes significativos y duraderos. Los docentes de Educación Física, suelen ser los grandes dinamizadores de los centros, y principalmente el diseño de actividades basadas en el juego, la expresión corporal, la danza, deportes y medio natural, pueden ser enfocadas perfectamente desde otras áreas. En E.I. no existe como tal elárea de Educación Física, pero en la mayoría de los centros los contenidos motrices de las áreas de E.I. son asumidas por maestros especialistas en Educación Física, y múltiples son la experiencias que han logrado resultados positivos en esta etapa desde el punto de vista de desarrollo de la motricidad, de los valores o de las actitudes (Barcala-Furelos, Carbia-Rodríguez, Peixoto-Pino, AbelairasGómez, \& Rodríguez-Núñez, 2017; Guimaraes, 2009; Teixeira-Costa, Abelairas-Gómez, Arufe-Giráldez, Pazos-Couto, \& Barcala-Furelos, 2015). Existen en la literatura experiencias basadas en la misma metodología, como por ejemplo un proyecto basado en Olimpismo (Peixoto-Pino, 2015a) en cuyo cronograma de actividades se basó nuestra propuesta, con resultados satisfactorios de cara a la adquisición de las competencias curriculares.

Durante el diseño de este proyecto se han tenido en cuenta las evidencias didácticas y científicas para la elaboración de contenidos curriculares, atendiendo a los nuevos retos de la educación. El uso de juegos motores relacionados ya han sido aplicados con éxito en diferentes niveles (Palacios-Aguilar, Del Castillo, Rodríguez, Gili, \& Del Gaiso, 2015). El aprendizaje mediante las nuevas tecnologías, concretamente mediante el visionado de vídeos puede favorecer la adquisición de conductas positivas y la viralización de su contenido en redes sociales, aportando un uso positivo de estos medios. La reflexión sobre la educación igualitaria y no sexista es algo en que los docentes deben prestar especial atención, favoreciendo la reflexión crítica para poder cambiar actitudes y creencias que no promueven la igualdad (MartínezMartínez, 2016).

Aprender conceptos básicos para la prevención y estabilizarlos en la memoria a largo plazo puede lograrse desde la escuela infantil. Un pequeño estudio piloto demostró que niños de 6 años son capaces de comprender los riesgos de playas y piscinas para la prevención del ahogamiento (Barcala-Furelos et al., 2017). En la educación primaria es un buen momento para aprender la reanimación cardiopulmonar, aunque su aplicación todavía sea muy rudimentaria y sin alcanzar los parámetros de calidad definidos en las recomendaciones internacionales (OteroAgra et al., 2018), el objetivo que promueven estos aprendizajes no es el de la correcta ejecución en edad escolar, sino el aprendizaje de actitudes y habilidades, para una buena aplicación en la vida adulta y aumentar así las tasas de testigos capaces de ayudar en caso de emergencia.

\section{Conclusiones}

\section{Implicaciones para la práctica docente.}

Este artículo pretende demostrar como un claustro de profesores, coordinado y con iniciativa pedagógica común puede organizar e implementar un proyecto interdisciplinar sobre una temática educativa curricularmente reconocida y socialmente necesaria. Es responsabilidad de toda la comunidad promover este tipo de acciones que repercutirán en un proceso de enseñanza/aprendizaje más reflexivo y eficiente.

\section{En relación a la evaluación del proyecto:}

- Aspectos conseguidos: con el presente proyecto se ha sensibilizado al alumnado, familias y sobre todo a los maestros, de la cantidad de peligros que nos rodean y la importancia de estar preparados para actuar con la mayor rapidez posible y de una manera idónea. Se mejoró, también el trabajo en grupo y la colaboración como clave del éxito (en los juegos y, lo que es más importante, en la propia supervivencia en caso de un accidente).

- Aspectos no conseguidos: No ha sido posible llegar a la totalidad del profesorado, del alumnado y de las familias. Por suerte, el grupo de gente que no ha participado ha sido muy reducido pero sí es cierto que no se consiguió sensibilizar a la comunidad educativa al completo.

- Aspectos mejorables: aunque la formación acerca de los Primeros Auxilios básicos se hizo de forma minuciosa con el profesorado (sobre todo respecto a la cadena básica de supervivencia y RCP), tal vez en las aulas quedó un poco reducido puesto que por el escaso tiempo cada grupo se centró en el tema que le correspondía para la elaboración del mural y no fue capaz de profundizar en el resto de contenidos.

\section{Líneas para futuros proyectos y experiencias} interdisciplinares

Como conflicto de intereses, mencionar que este proyecto fue presentado por primera vez y en formato de conferencia, para discusión pública en el en Congreso Internacional de Seguridad y Turismo Sostenible. VIII de Socorrismo Basado en la Evidencia Científica(PeixotoPino, 2015b), y de la discusión con expertos y sus aportes se elaboró la experiencia en formato de artículo para divulgar a la totalidad de la comunidad científica, además de la evaluación docente se proponen las siguientes líneas de futuro:

- Ampliación del proyecto a otros centros del entorno.

- Estudio longitudinal de las consecuencias e implicaciones 
pedagógicas en relación con la prevención de accidentes.

- Implementación del proyecto en la educación secundaria o la repetición cada dos años de actividades encaminadas a perpetuar las habilidades y las actitudes.

- Solicitud a los centros de formación del profesorado de recursos y cursos para docentes sobre esta temática.

\section{Referencias}

Barcala-Furelos, R., Carbia-Rodríguez, P., Peixoto-Pino, L., AbelairasGómez, C., \& Rodríguez-Núñez, A. (2017). Implantación de programas educativos para prevenir ahogamientos. ¿Qué se puede hacer desde la escuela infantil? Medicina Intensiva. https://doi.org/ 10.1016/j.medin.2017.08.005

Bohn, A., Lukas, R. P., Breckwoldt, J., Böttiger, B. W., \& VanAken, H. (2015). «Kids save lives»: why schoolchildren should train in cardiopulmonary resuscitation. Current Opinion in Critical Care, 21(3), 220-225. https://doi.org/10.1097/MCC.0000000000000204

Castellar, C., Pradas, F., Rapún, M., Coll, I., \& Pérez, S. (2013). Aula en bici: un proyecto longitudinal de intervención docenteen Ed. Primaria (Class by Bicycle: a longitudinal project of educational intervention in primary school). Retos. Nuevas tendencias en Educación Física, Deporte y Recreación, 23, 5-9.

Díaz, J. D. (2010). Educación física e interdisciplinaridad: una relación cada vez más necesaria. Tándem: Didáctica de la educación física, 33, 7-21.

Gómez, A., Díez, L. J., Fernández, J. M., Gorrín, A., Pacheco, J.J., \& Sosa, G. (2008). Nueva propuesta curricular para el área de Educación Física en la Educación Primaria. Revista Internacional de Medicina y Ciencias de la Actividad Física y del Deporte, 8(29), 93-108.

Greif, R., Lockey, A. S., Conaghan, P., Lippert, A., De Vries, W., Monsieurs, K. G, \& Collaborators. (2015). European Resuscitation Council Guidelines for Resuscitation 2015: Section 10. Education and implementation of resuscitation. Resuscitation, 95, 288-301. https://doi.org/10.1016/j.resuscitation.2015.07.032

Guimaraes, R. (2009). ¿Qué puede aportar la literatura infantil a una Educación Física para la paz? La Peonza: Revista de Educación Física para la paz, (4), 87-97.

Jorquera, J. L., Leiva, A., Molina, J.M., \& Sánchez,A. (2018). Proyecto educativo Olimpízate: objetivos, metodología y actividades para enseñar olimpismo en los centros de educación secundaria (Olimpízate, an educational project: objectives, methodology and activities to teach Olympism in secondary schools). Retos. Nuevas tendencias en Educación Física, Deporte y Recreación, 33, 286292.

Lago-Ballesteros, J., Basanta-Camiño, S., \& Navarro-Patón, R. (2018). La enseñanza de los primeros auxilios en educación física: revisión sistemática acerca de los materiales para su implementación. Retos. Nuevas tendencias en Educación Física, Deporte y Recreación,34, 349-355.

Ley Orgánica 2/2006, de 3 de mayo, de Educación. Boletín Oficial del Estado, 106. Recuperado de https://www.boe.es/buscar/pdf/2006/ BOE-A-2006-7899-consolidado.pdf

Martínez-Martínez,A. (2016). La co-educación en el ámbito educativo/ :proyecto de intervención interdisciplinar en la materia de educación física. Recuperado de http://repositori.uji.es/xmlui/handle/10234/ 167223

Otero-Agra, M., Rodríguez-Núñez, A., Rey, E., Abelairas-Gómez, C., Besada-Saavedra, I., Antón-Ogando, A. P., \& Barcala-Furelos, R. (2018). What biomechanical factors are more important in compression depth for children lifesavers? A randomized crossover study.Article in Press. https://doi.org/10.1016/j.ajem.2018.05.003

Palacios-Aguilar, J., Del Castillo, M., Rodríguez, J. E., Gili, C.M., \& Del Gaiso, M. (2015). Juegos aplicados al socorrismo en el área de educación física. EmásF: revista digital de educación física, 35, 3657.

Peixoto-Pino, L. (2015a). Proyecto interdisciplinar, «escuela: comunidad olímpica». Retos. Nuevas tendencias en Educación Física, Deporte y Recreación, 25, 140-143.

Peixoto-Pino, L. (2015b). Proyecto interdisciplinar: Tú puedes salvar una vida. En Congreso Internacional de Seguridad y Turismo Sostenible. VIII de Socorrismo Basado en la Evidencia Científica, 1, 10. Oleiros (A Coruña): Universidad de A Coruña.

Pichel-López, M., Martínez-Isasi, S., Barcala-Furelos, R., FernándezMéndez, F., Vázquez Santamariña, D., Sánchez-Santos, L., \& Rodríguez-Nuñez, A. (2017). Un primer paso en la enseñanza del soporte vital básico en las escuelas: la formación de los profesores. Anales de Pediatría. https://doi.org/10.1016/j.anpedi.2017.11.002

Proxecto educativo E Por Qué? (s.f.). Recuperado de https:// www.edu.xunta.es/espazoAbalar/sites/espazoAbalar/files/datos/ 1303196843/contido/e_por_que/eporque2.html

Real Decreto 1630/2006, de 29 de diciembre, por el que se establecen las enseñanzas mínimas del segundo ciclo de Educación infantil.(s.f.). Recuperado de http://noticias.juridicas.com/base_datos/Admin/ rd1630-2006.html

Real Decreto 126/2014, de 28 de febrero, por el que se establece el currículo básico de la Educación Primaria. (s.f.). Recuperado de http://noticias.juridicas.com/base_datos/Admin/524514-rd-1262014-de-28-feb-establece-el-curriculo-basico-de-la-educacionprimaria.html

Teixeira-Costa, H., Abelairas-Gómez, C., Arufe-Giráldez, V., PazosCouto, J. M., \& Barcala-Furelos, R. (2015). Influence of a physical education plan on psychomotor development profiles of preschool children. Journal of Human Sport and Exercise, 10(1), 126-140.

World Health Organization (2008). World Report on Child Injury Prevention. Geneva: World Health Organization.
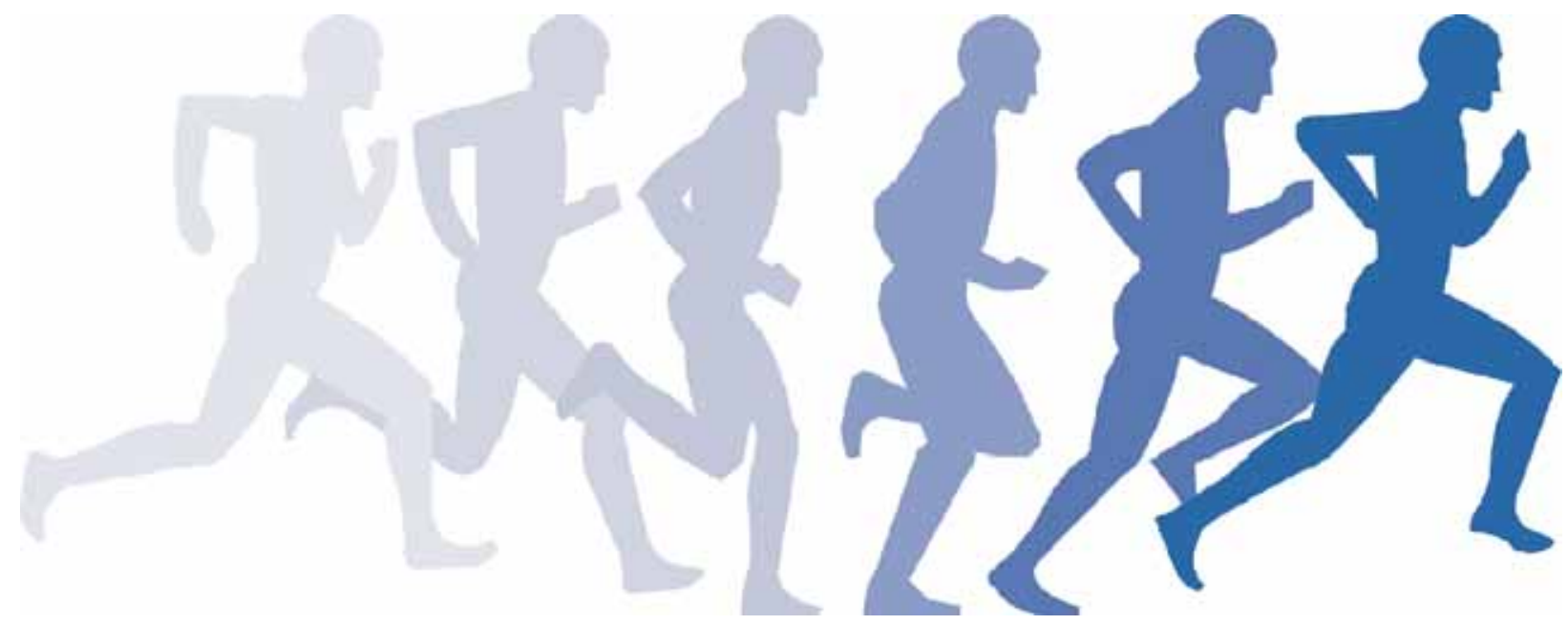